\title{
Characterization of horizontal flows around solar pores from high-resolution time series of images
}

\author{
S. Vargas Domínguez ${ }^{1}$, A. de Vicente ${ }^{2}$, J. A. Bonet ${ }^{2}$, and V. Martínez Pillet ${ }^{2}$ \\ ${ }^{1}$ Mullard Space Science Laboratory, University College London, Holmbury, St Mary, Dorking, RH5 6NT, UK \\ e-mail: svd@mssl.ucl.ac.uk \\ 2 Instituto de Astrofísica de Canarias, 38205 La Laguna, Tenerife, Spain
}

Received 8 September 2009 / Accepted 8 March 2010

\section{ABSTRACT}

\begin{abstract}
Context. Though there is increasing evidence linking the moat flow and the Evershed flow along the penumbral filaments, there is not a clear consensus regarding the existence of a moat flow around umbral cores and pores, and the debate is still open. Solar pores appear to be a suitable scenario to test the moat-penumbra relation as they correspond to a direct interaction between the umbra and the convective plasma in the surrounding photosphere without any intermediate structure in between.

Aims. We study solar pores based on high-resolution ground-based and satellite observations.

Methods. Local correlation tracking techniques were applied to different-duration time series to analyze the horizontal flows around several solar pores.

Results. Our results establish that the flows calculated from different solar pore observations are coherent among each other and show the determining and overall influence of exploding events in the granulation around the pores. We do not find any sign of moat-like flows surrounding solar pores, but a clearly defined region of inflows surrounding them.

Conclusions. The connection between moat flows and flows associated to penumbral filaments is hereby reinforced.
\end{abstract}

Key words. Sun: activity - Sun: photosphere - Sun: granulation

\section{Introduction}

The solar photosphere displays a wide variety of magnetic features at different spatial scales, with sunspots and pores the more conspicuous ones. The morphology and evolution of sunspots and pores have been extensively studied and both are thought to be ruled by the mutual effects of magnetic fields that inhibits convection (though not completely) and plasma motions. Nevertheless, there is no clear consensus for a model explaining the transition from pores into sunspots (i.e. development of penumbra, see the monograph by Thomas \& Weiss 2008), the fading of the Evershed flow once it leaves the penumbra and enters the region dominated by the large outflows (the so-called moat flows), the flow patterns surrounding pores which are alternatively dominated by downflows surrounding them (Giordano et al. 2008) or moat-like flows (Zuccarello et al. 2009) and also the effect of the penumbra in the granular pattern surrounding sunspots.

By using local correlation tracking techniques Vargas Domínguez et al. (2007) found a direct correlation between penumbrae and the appeareance of moat flows in a complex $\delta$-configuration active region. A more extensive sample taking into account different penumbral configurations was analyzed by Vargas Domínguez et al. (2008), establishing a systematic moat-penumbra relation in all sunspots under study. According to these studies, no moat flow was detected in the granulation next to umbral boundaries without penumbrae. Moat flows were always detected as a prolongation of the penumbral filaments once they crossed the penumbral boundary.

Though there is increasing evidence linking the moat flow and the Evershed flow along the penumbral filaments (e.g. Sainz Dalda \& Martínez Pillet 2005; Cabrera Solana et al. 2006), the debate regarding the existence of a moat flow around umbral boundaries without penumbra and individual pores is still ongoing. In a recent work, Deng et al. (2007) found that the dividing line between radial inward and outward proper motions in the inner and outer penumbra respectively survived the decay phase, suggesting that the moat flow is still detectable after the penumbra disappeared. However, previous works (Sobotka et al. 1999; Roudier et al. 2002; Hirzberger 2003) measured horizontal proper motions in and around pores and observed some penetrating flows at the umbral boundaries as well as a ringlike arrangement of positive divergences (rosettas) around the pores, which is related to a continuous activity of exploding granules in the granulation around them. Roudier et al. (2002) identified a very clear inflow around pores, which corresponds to the penetration of small granules and granular fragments from the photosphere into the pores, pushed by granular motions that originate in the divergence centers around them. These authors conclude that the motions at the periphery of the pore are substantially and continuously influenced by the external plasma flows deposited by the exploding granules. It is important to note in this context that the annular area surrounding pores and filled with exploding granules generates an outward directed flow annulus that can give the impression of a persistent outflow.

Converging flows around pores have also been observationally reported previously by Wang \& Zirin (1992), as have downflows at their periphery (Sankarasubramanian \& Rimmele 2003). More recently, 3D magnetohydrodynamic simulations found horizontal flows towards the pores that contribute to mantain their magnetic structure (Cameron et al. 2007).

Pores are interesting to analyze because, as they do not display penumbrae (Keil 1999), what we actually observe is the 
A\&A 516, A91 (2010)

Table 1. Characteristics of the time series of solar pores observed from ground-based and satellite facilities.

\begin{tabular}{cccccccc}
\hline \hline Telescope & Date 2007 & Series & Time, UT & Duration [min] & N. images & Cadence [s] & FOV ["] \\
\hline \multirow{2}{*}{ SST } & \multirow{2}{*}{ Sep. 30 } & 1 & $08: 43-09: 31$ & 48 & 286 & 10 & $64.3 \times 65.0$ \\
& & 2 & $09: 36-09: 56$ & 20 & 118 & 10 & $64.3 \times 65.0$ \\
\hline \multirow{3}{*}{ Hinode } & \multirow{2}{*}{ Jun. 1 } & 1 & $21: 35-21: 55$ & 20 & 40 & 30 & $27.9 \times 55.7$ \\
\cline { 2 - 8 } & \multirow{2}{*}{ Sep. 30 } & 1 & $22: 26-23: 33$ & 67 & 134 & 30 & $27.9 \times 55.7$ \\
\hline
\end{tabular}

direct interaction between the umbra (with a strong vertical magnetic field that inhibits convection inside it) and the convective plasma in the surrounding photosphere without any intermediate structure in between. Many observed features like bright granules moving in the border of a pore (Sobotka et al. 1999) show the complex exchange taking place between the pore and its surrounding granulation.

Our main interest is the characterization of the horizontal flows around a variety of solar pores on the basis of a highresolution time series of images. Observations from groundbased and space telescopes are analyzed by means of the local correlation tracking technique. In Sect. 2 the paper concentrates separately on the description of the image acquisition and data processing for ground-based and satellite data. The analysis of the data and the presentation of results are treated in Sect. 3. A general summary and final discussion are presented in Sect. 4.

\section{Observations and data processing}

A significant part of our data were acquired during a long observing campaign (24 days) carried out in September-October 2007 with the cooperation of several European and Japanese institutions and joint observations from several solar telescopes of the Canary Islands Observatories: SST and DOT in La Palma and VTT and THEMIS in Tenerife. Moreover, and for the very first time, coordinated observations with the space solar telescope Hinode (Kosugi et al. 2007) were performed in the framework of the Hinode Operation Program 14. In the next sections we will detail the ground-based and satellite observations supporting the present work as well as the specific data processing applied in each case.

\subsection{Ground-based SST data}

The data from the Swedish 1-m Solar Telescope (SST, Scharmer et al. 2003a,b) analyzed here were recorded during a particular observing run on 2007 September 30 and correspond to the active region NOAA 10971. The main target was a region close to the solar disk center $(\mu=0.98)$ with some pores of different sizes embedded in a plage region that exhibits an intense magnetic activity.

A dichroic beam-splitter in the optical setup divided the light beam into two channels: blue and red. Images in G-band ( $\lambda 4305.6 \AA)$ were acquired in the blue beam at a rate of 13 frames s$^{-1}$. We used 12 bit detectors of $2048 \times 2048$ square pixels, which in combination with an image-scale of 0.034 arcsec/pix rendered an effective field-of-view (hereafter FOV) of $69 \times 69$ square arcsec. After dark-current subtraction and flatfielding, the G-band images were corrected for atmospheric and instrumental degradation by employing the so-called multi-frame blind-deconvolution restoration technique (MFBD, Löfdahl 1996, 2002). For each wavelength, the image sequence was grouped in sets of about 80 consecutive frames acquired within time intervals of $10 \mathrm{~s}$ each. Every set yielded one restored image. The next steps in the data post-processing were the compensation for diurnal field rotation, rigid alignment of the images, correction for distortion and finally subsonic filtering. For more details see Vargas Domínguez (2008).

The red beam fed the filter Solar Optical Universal Polarimeter (SOUP, Title et al. 1986) to obtain H $\alpha(\lambda 6562.8 \AA)$ and narrow-band images at $6302 \AA$. Single images were taken at a rate of 35 frames s$^{-1}$ with CCDs of $1024 \times 1024$ square pixels and an image-scale of 0.065 arcsec/pix. A beam splitter in front of the SOUP deflected a fraction of light to obtain simultaneous broad-band phase diversity image-pairs in the continuum near $\lambda 6302 \AA$. These images made up an additional object for the multi-object multi-frame blind-deconvolution (MOMFBD, Van Noort et al. 2005) algorithm to jointly restore both the broad-band and the narrow-band images. From the restored narrow-band images we computed longitudinal magnetograms and dopplergrams.

Due to periods of bad seeing, in which the quality did not reach the desired top level, some of the images were discarded and we kept only the best and longest consecutive sequence of images. The very final product were two G-band time series with the characteristics listed in Table 1. The time gap of about $5 \mathrm{~min}$ between the two consecutive series for each wavelength resulted from a telescope tracking interruption. That is also the reason why the FOV is slightly different in the time series. Most images forming the time series show details near the diffraction limit of the telescope.

The procedure followed for image restoration is extensively detailed in Vargas Domínguez (2008). Figure 1 shows one of the co-temporal sets of images of the emerging active region after restorations. The upper left panel shows the FOV covered by the observations displaying the pores under analysis, and the other panels give useful information to characterize the region as derived from the red channel simultaneous observations.

\subsection{Hinode space satellite data}

After its launch on 2006 September 22, Hinode has become an extremely successful observatory for Solar Physics research. It has managed to observe many high-detailed solar features by avoiding the blurring and distortion effects produced by the Earth's atmosphere. The public archive of Hinode ${ }^{1}$ is an organized data base where all Hinode observations can be found out and easily downloaded.

We were interested in Hinode observations of solar pores taken with the Solar Optical Telescope (SOT, Tsuneta et al. 2008) to pursue our study of photospheric horizontal flows. The next two sections describe the Hinode data we analyze in this paper. These data cover the sample of cases studied here and

\footnotetext{
1 See the website: http://solar-b.nao.ac.jp/hsc_e/darts_e. shtml
} 

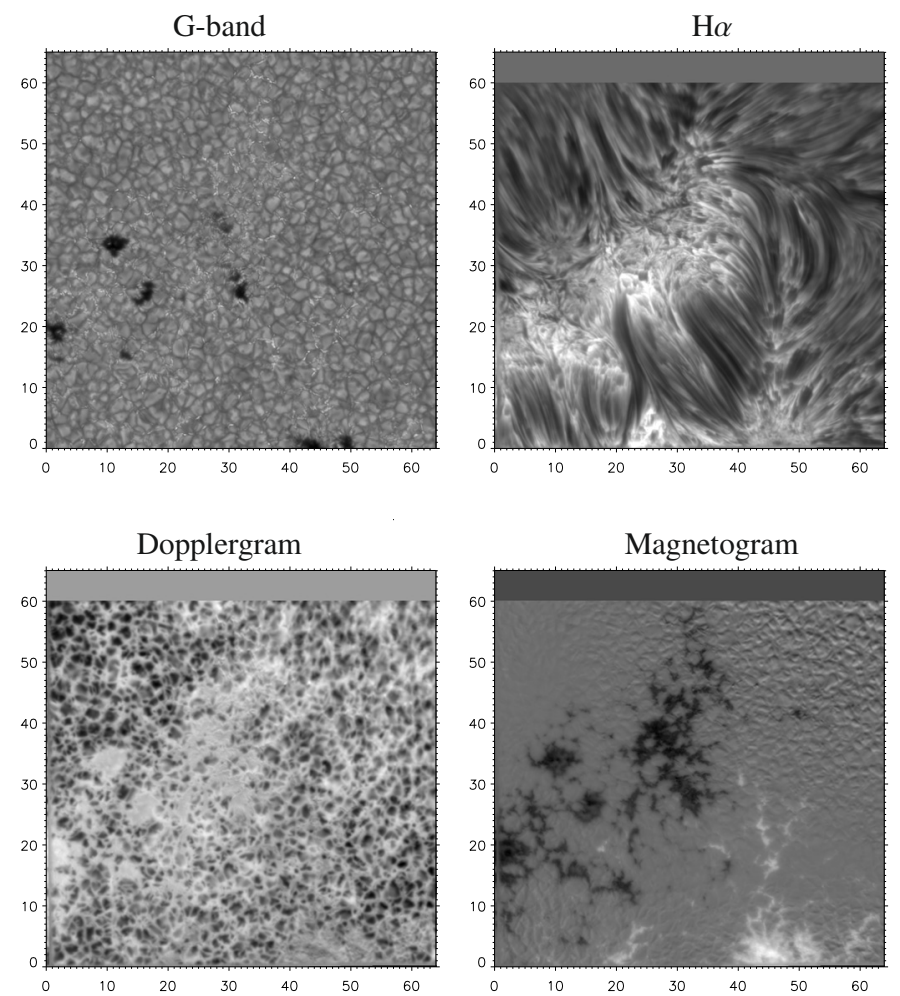

Fig. 1. SST co-temporal and co-spatial set of images from the emerging flux region on 2007 September 30. Dark regions in the Dopplergram denote upflows, and the map ranges from approx. -1.2 (white) to 1.6 (black) $\mathrm{km} \mathrm{s}^{-1}$. The LOS magnetogram shows negative/positive (black/white) polarities with field strengths up to $\sim 2300 \mathrm{G}$. The coordinates are expressed in arcsec.

moreover give us the possibility to compare them with the results from ground-based observations.

\subsubsection{Data from 2007 June 1}

These data correspond to a solar portion including an isolated and round-shaped pore observed by Hinode on 2007 June 1. Images were acquired in G-band with a cadence of $30 \mathrm{sec}$ in a solar portion close to the disk center $(\mu=0.87)$. After subsonic filtering we obtained two time series; Table 1 (Jun. 1) summarizes the parameters of both series in detail. The treatment performed on Hinode data does not include any restoration process because they were not degraded by atmospheric turbulence.

\subsubsection{Data from 2007 September 30}

The data from Hinode on 2007 September 30 correspond to the coordinated observations described in Sect. 2.1. Hinode observed the emerging flux region NOAA 10971 in G-band from 00:14 to 17:59 UT with a few brief interruptions for calibrations. Table 1 (30 Sep.) summarizes the parameters of the time series in more detail. A significant part of the FOV in the SST images is covered by the Hinode observations.

Hinode data often presents misalignments due to tiny tracking flaws and also due to temporal interruptions. We proceeded by aligning all of the 1030 images (18 h) at a sub-pixel level. Subsonic filtering was applied to eliminate residual jittering in the time series.

\section{Data analysis and results}

\subsection{General description of horizontal proper motions in the FOV}

The G-band series taken at both SST and Hinode were employed to analyze the horizontal proper motions of structures in every FOV by using the local correlation tracking (LCT) technique (November \& Simon 1988) as implemented by Molowny-Horas \& Yi (1994). This technique works by selecting small subfields around the same pixel in contiguous snapshots, which are correlated to find the best match-displacement. The procedure provides a map of displacements or proper motions per time step (i.e. velocities), which we average in time. The subfields to be correlated are defined by a Gaussian window whose full-width at half-maximum $(F W H M)$ is set according to the size of the structures we want to track. In this section we show the maps of horizontal velocities calculated for the different time series by using a Gaussian tracking window of FWHM 1.'0, which is roughly half of the typical granular size.

Figure 2 shows the flow maps computed from the two restored time series from SST. The velocities were averaged over the total duration of each series. The underlying background in the maps is the average image of the respective series. As commented on above, the FOV is slightly different in both cases so that for instance the pore at coordinates $\left[17^{\prime \prime}, 26^{\prime \prime}\right]$ in the upper map of the figure is located at $\left[24^{\prime \prime}, 42^{\prime \prime}\right]$ in the lower map.

The maps are dominated by flows coming from exploding events taking place all over the FOV. As expected, the map averaging over a longer time period (48 min) is slightly smoother than the other one (20 min) and displays lower velocities. Nevertheless, both maps reproduce similar flow patterns all over the FOV. The top of the FOV in Fig. 2 (upper panel) shows very conspicuous exploding granular events, which are grouped at every upper corner of the FOV forming two large-scale structures that fit the supergranular one well. Interestingly, there is an evident anti-correlation between the strength of the horizontal velocity field and the mean magnetic flux density in those locations as shown in the co-temporal and -spatial magnetogram in Fig. 1. A smaller portion of these structures can also be identified in the lower panel of Fig. 2.

Even though a complete description of the proper motions around the pores in the FOV will be done in Sect. 3.5, a glance at the figure reveals no evidence of a moat-like pattern around any of the pores. The central part of the FOV where the smaller pores are embedded exhibits a lower magnitude of horizontal velocities. This behavior is explained by the intense magnetic activity in this part of the FOV as unveiled by the corresponding magnetogram of the zone shown in Fig. 1.

Concerning Hinode data, the computed map of horizontal velocities for the time series of 2007 June 1 is displayed in Fig. 3. This map is calculated from the longest time series $(67 \mathrm{~min}$, see Table 1); the analysis of the shortest series (not presented here) gives similar results, although as expected, noise and velocity magnitudes increase significantly. The isolated solar pore is immersed in a granular region displaying several and recurrent large exploding granular events all around it. This velocity map as well as those derived from the SST data lead us to conclude that an imaginary line connecting the centers of the exploding events (i.e. mesogranules) around a pore outlines a round-shaped contour at a distance from the pore border comparable to the pore diameter. Velocity magnitudes in the outer part of the outlined contour are clearly larger than those in the inner part between the contour and the pore border. The resulting map of horizontal velocities for 2007 September 30 data will be 

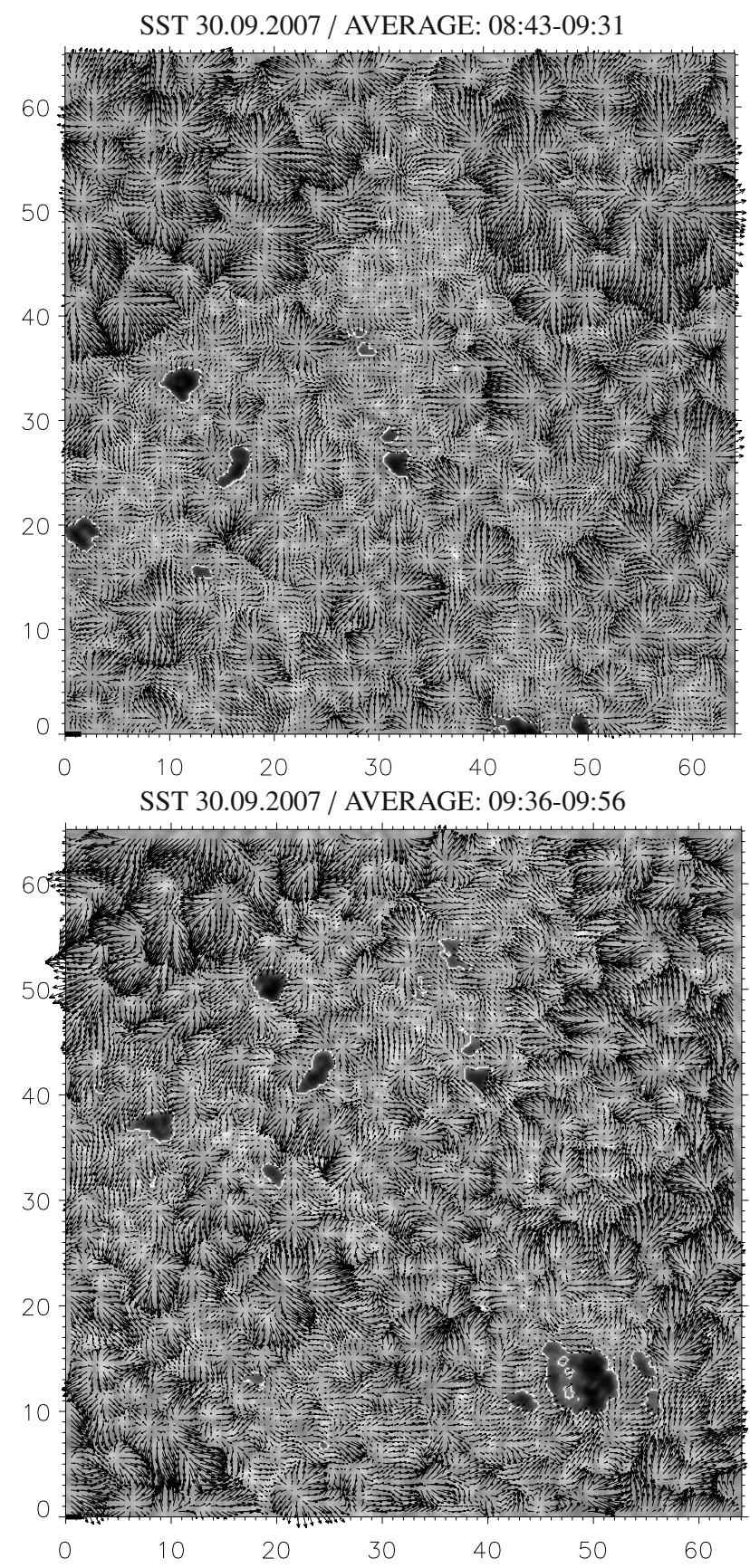

Fig. 2. Map of horizontal velocities ( FWHM 1". 0 ) from the restored time series taken at the SST on 2007 September 30. The velocities are averaged over $48 \mathrm{~min}$ (upper panel) and $20 \mathrm{~min}$ (lower panel). Arrows show the direction of horizontal velocities and their length the correspondent absolute value in all flow maps hereafter. The white contours outline the border of solar pores. The length of the black bar at coordinates $(0,0)$ corresponds to $1.6 \mathrm{~km} \mathrm{~s}^{-1}$. The coordinates are expressed in arcsec. The background in every case represents the average image of the corresponding G-band series.

presented in Sect. 3.3, where we pursue a detailed analysis for this time series.

\subsection{Averaging horizontal flows within different temporal windows}

To study the influence of different time averages in the velocity maps we considered temporal windows of 5, 10, 15 and $20 \mathrm{~min}$
Hinode 01.06.2007 / AVERAGE: 22:26-23:33

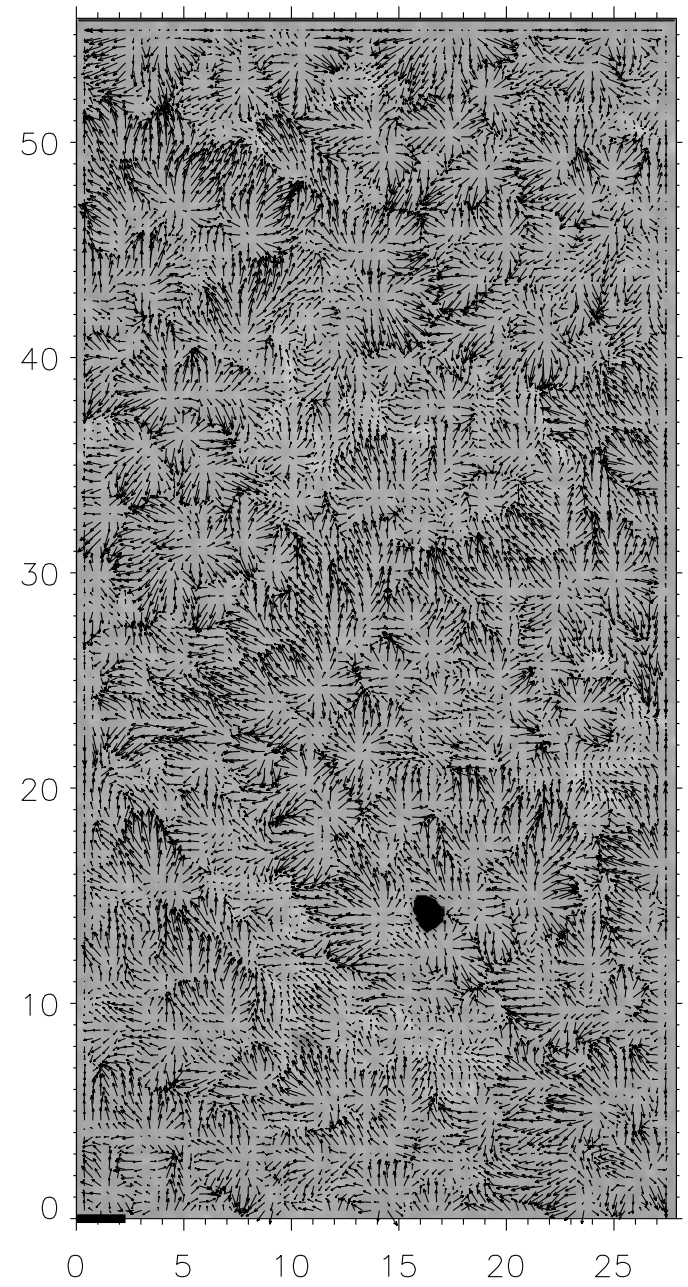

Fig. 3. Maps of horizontal velocities ( $F W H M$ 1', 0,67 min average) for the processed time series taken by Hinode on 2007 June 1 . The length of the black bar at coordinates $(0,0)$ corresponds to $2.3 \mathrm{~km} \mathrm{~s}^{-1}$. The coordinates are expressed in arcsec. The background represents the average image of the G-band series.

in the best quality G-band time series recorded at the SST (series 2 of September 30 in Table 1). As expected, the maps are smoother for longer averaging periods. The two extreme cases are shown in Fig. 4, displaying a close-up at some of the pores in the left part of the FOV in Fig. 2 (lower panel). Figure 5 shows the histograms of the velocity magnitudes for each averaging temporal window considered. The resulting distribution is very similar in the four cases, but the histograms shift to the left as the averaging period increases. Thus the largest velocity magnitudes range from 1.83 to $1.97 \mathrm{~km} \mathrm{~s}^{-1}$ and the mean values from 0.48 to $0.50 \mathrm{~km} \mathrm{~s}^{-1}$. In both cases, smaller/larger values correspond to longer/shorter time averages.

Regardless of whether we averaged over 5 or $20 \mathrm{~min}$ intervals, we found essentially the same general trends with exploding granular patterns all over the FOV, meaning that the averaging periods we are employing are smaller than or about the lifetime of the observed structures. We do not recognize any moat-like flow around the pores for such different time averages as shown in Fig. 4. 


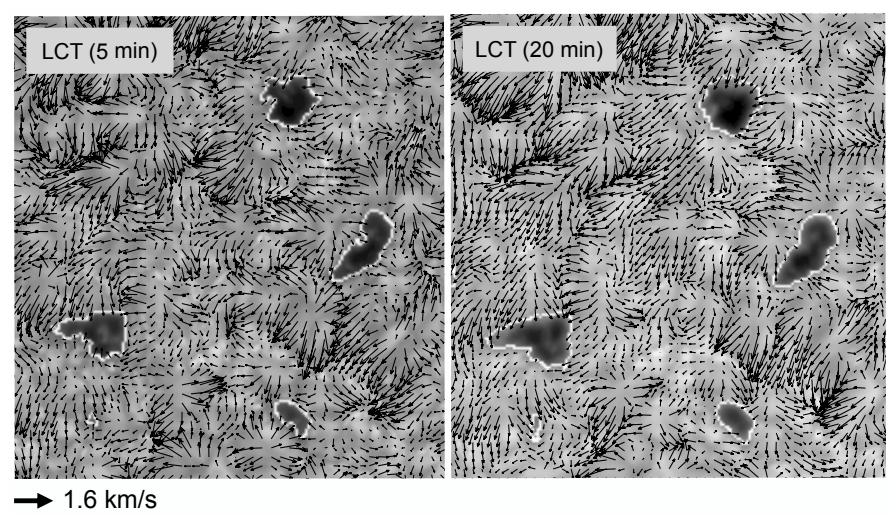

Fig. 4. Maps of horizontal velocities (FWHM 1.'0) for the SST time series on 2007 September 30. Velocities are derived from LCT for two different time averages as labeled. The background in every case represents the average image of the corresponding G-band series. The FOV of every image is $25^{\prime \prime} \times 27^{\prime \prime}$.

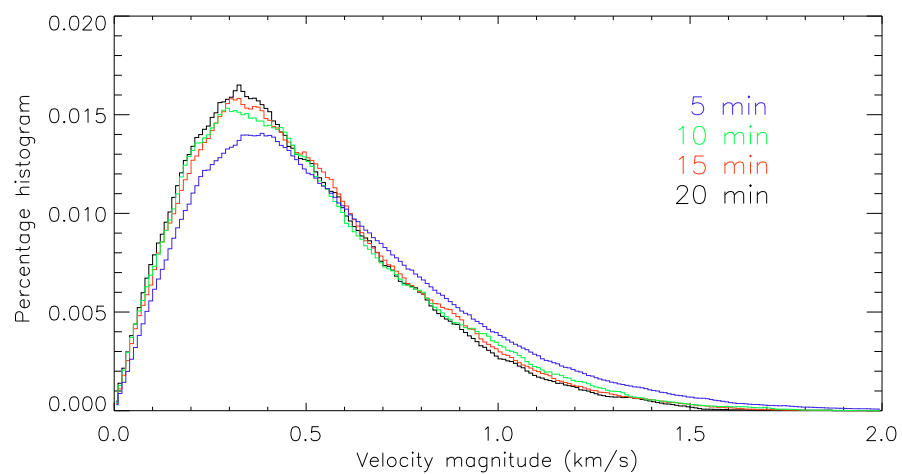

Fig. 5. Histogram of horizontal velocity magnitudes for different time intervals. A local correlation tracking technique was used to derive the horizontal velocities $\left(F W H M=1{ }^{\prime \prime} 0\right)$.

\subsection{Long-term evolution of the velocity field}

In order to investigate how the evolution of the emerging region affects the velocity in the FOV for long periods of time, particularly around solar pores, we used the Hinode time series of 2007 September 30, which corresponds to $18 \mathrm{~h}$ of almost continuous solar observation as reported in Sect. 2.2.2. The stable good quality and long duration of the series is ideal to study the evolution of the flow maps in an emerging flux region. For this purpose the images were grouped in 1-h sets from 00:14 UT up to the time 14:00 UT (excepting the first set spanning from 00:14 UT to 00:59 UT), and every set was processed independently which resulted in 14 maps of horizontal velocities, which are computed by LCT using a Gaussian tracking window of FWHM 1". 0 and 1-h averages. Figure 6 shows out of these fourteen maps those starting at 00:14 UT, 05:00 UT, 09:00 UT and 13:00 UT, respectively. A movie displaying the evolution of the pore throughout the 18-h observation can be downloaded from the website http://www.iac.es/proyecto/solarhr/ hinode30sep2007. mov.

The main pore is located in the spatial position [37", 55"] in the first map (Fig. 6) and surrounded by some smaller pores, which form a sort of vertical and elongated arrangement in the figure. The collection of these pores is evolving in time, and some of them start merging and disappearing. The final picture of the region displayed in Fig. 6 shows the isolated main pore with only a very tiny magnetic companion.
We could not identify any signal of a moat-like flow around the pores in any of the evolutionary stages shown in the maps sequence, but found continuous activity caused by exploding granules. Centers of divergence were systematically identified, some of them very close to the pore border. Proper motions displaying inward components are more common around the pores and no outward regular large-scale flow corresponding to a moat-flow was found. These results, the reliability of which is supported by the long duration of the sample studied, reinforce the previous analysis pursued for the only 1-h long SST data in Sect. 3.1.

\subsection{Distribution of horizontal speeds in the FOV}

Figure 7 shows the location (white areas) of speeds (velocity magnitudes) within three different ranges in $\mathrm{km} \mathrm{s}^{-1}$ for the SST data (the first series in Table 1): low velocity magnitudes lower than 0.3 , medium velocity magnitudes in the range $0.3-0.8$ and high velocity magnitudes greater than 0.8 .

Small speeds are mainly grouped in the central part of the FOV, where an intense magnetic activity is detected as evidenced by the high concentration of G-band bright points and faculae present in this region (see Fig. 1). Around the pores the velocity magnitudes mainly correspond to the lower range $\left(<0.3 \mathrm{~km} \mathrm{~s}^{-1}\right)$ so that they are surrounded by white areas in Fig. 7 (upper panel). The areas mapping medium velocity magnitudes are regularly spread out all over the FOV except in the proximity of pores as shown in Fig. 7 (middle panel). High velocities $\left(>0.8 \mathrm{~km} \mathrm{~s}^{-1}\right)$ are not homogeneously distributed in the FOV, but are mainly grouped at the two upper corners of Fig. 7 (lower panel). The magnetogram in the last panel of Fig. 1 has been overlapped to better identify the match between the locations displaying higher velocity magnitudes and the less-magnetized regions (scaled as 0 and colored in light blue). These large flows zones might reflect supergranular cells as mentioned in Sect. 3.1.

\subsection{Velocity distribution around solar pores}

From the computed velocity fields we can perform a detailed analysis of the velocity distribution around the solar pores. For this we will use the region observed with the SST where we have a useful collection of pores available to work with. As we have two time series for this region (see Table 1), we will employ the first one covering the longest time period, except for one of the pores which is out of the FOV. For this pore we will use the second time series. Figure 8 shows the FOV including all the pores under study, which are labeled with consecutive numbers for easy identification hereafter.

Figure 8 (lower panel) illustrates the projection of the velocities into radial and transversal components as a convenient way to compute inward and outward motions. The figure plots two points in the granulation surrounding a solar pore with their corresponding velocity vectors $\boldsymbol{v}$. The pore is centered at coordinates $\left(x_{\mathrm{c}}, y_{\mathrm{c}}\right)$ with respect to the orthogonal coordinate system $X, Y$ placed at the lower left corner of the FOV. Vector $r$ is the position vector of a given point with respect to the pore center. The pore center is located at its gravity-center calculated by weighting the position of every point inside the pore with the inverse of its respective intensity. Velocity vectors in every point of the granulation surrounding the pore are projected into radial $v_{r}$ and transversal $v_{t}$ components.

In order to establish the inward and outward motions, we first selected the FOV including the pore under study. Because active regions in general and pores in particular exhibit their own 

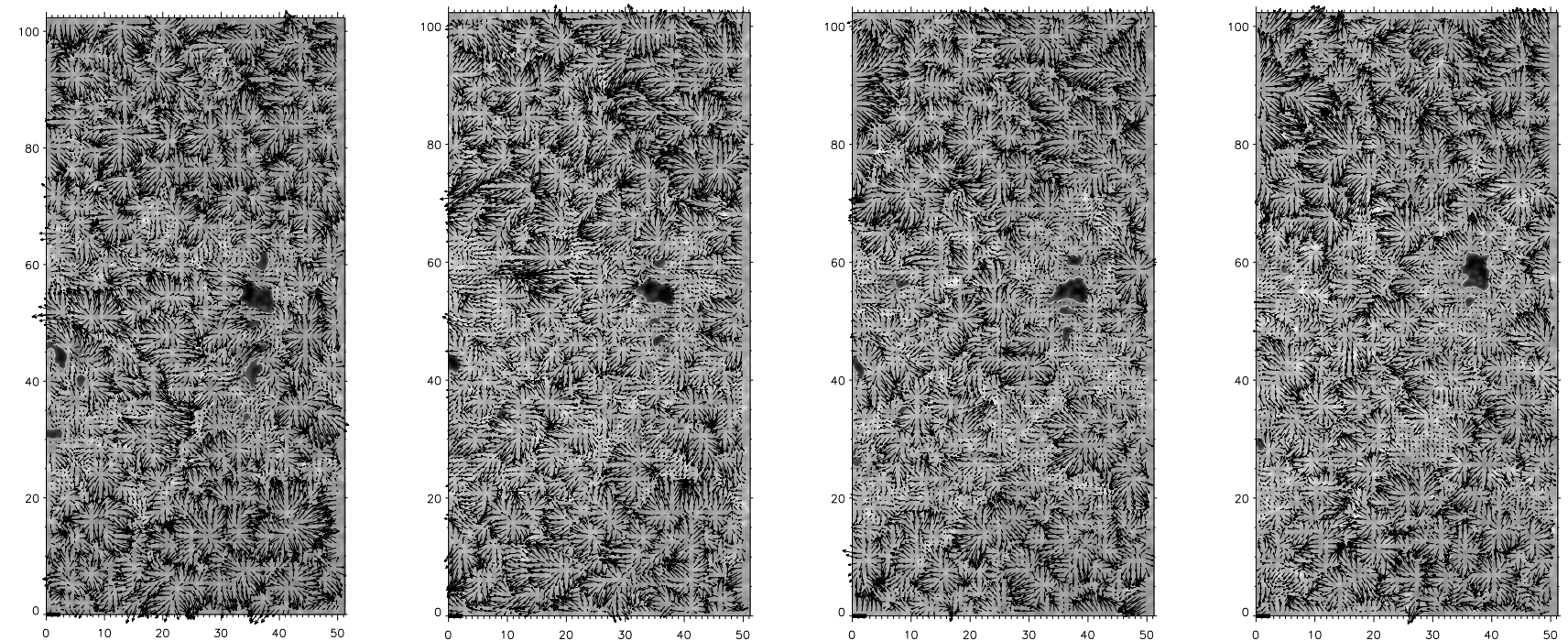

Fig. 6. Map of horizontal velocities (FWHM 1.'0) in the region displaying solar pores observed with Hinode on 2007 September 30 . Fourteen maps are produced, each one computed from data in 1-h intervals. From left to right the maps corresponding to intervals 1, 5, 9 and 13 respectively are shown (see the text for details). The length of the black bar at coordinates $(0,0)$ corresponds to $2.3 \mathrm{~km} \mathrm{~s}^{-1}$. The coordinates are expressed in arcsec. The background represents the average image in every hour-set.

displacement (due to differential rotation and intrinsic motions) while embedded in the granulation pattern, we aligned the time series with respect to an area framing the pore (correlation box) so that we made sure we were measuring plasma motions with respect to the pore. We computed the map of horizontal velocities by LCT with the same Gaussian tracking window (FWHM $\left.1{ }^{\prime \prime} 0\right)$ employed in the previous sections. The next step was to define the "radial directions" (see lower panel in Fig. 8) that would be used as the reference to project the velocities. For short distances these directions are defined as perpendicular to the pore border. These perpendicular directions are calculated from the gradients of intensity in a smoothed pore-mask image ${ }^{2}$. This way one can also deal with non-round-shaped pores. The limit for short distances is defined by thresholding the intensity gradients. The threshold depends on the pore shape and size. At large distances (i.e. $\sim 3$ times larger than the pore mean radius) all pores are considered as round-shaped structures and the radial directions are defined by the position vector $\boldsymbol{r}$ of a given point with respect to the pore center. According to the lower panel in Fig. 8, inward/outward motions correspond to $\cos \beta$ negative/positive, where $\beta$ is the angle formed by $\boldsymbol{v}$ and the positive radial direction (outward) at each point of the FOV. The value of $\cos \beta$ is mapped in gray scale ranging from 1 for purely radial outward velocities (in black) to -1 for purely inward velocities (in white). A binary mask is created from the previous gray-scaled map where areas in black and white correspond to velocities with positive (outward) and negative (inward) radial components, respectively.

We applied the aforementioned method to our sample of seven different pores shown in the upper panel of Fig. 8. The results are displayed in Fig. 9, where rows display the different pores and the columns correspond to 1) the averaged FOV around the pore; 2) the velocity field; 3 ) the gray-scale representation of $\cos \beta$; and 4 ) the final binary representation mapping

\footnotetext{
2 A binary mask setting the area occupied by the pore $(1 / 0$ inside/outside the pore) is defined. Then this mask is smoothed by convolving with a Gaussian function so that we obtained a distribution of pseudo-intensities smoothly ranging from 1 to 0 and describing a blurred shape of the pore. This is the smoothed pore-mask image to which we refer above.
}

the areas with inward (white) and outward (black) radial velocity components after applying the binary mask. The analysis of all cases establishes that the flows display a clear preference for inward directions around the pores. This is systematically found in all examples. The more regular-shaped pores are surrounded by an also regular annular-like area with inward velocity components with a mean width similar to the center-to-border distance in the pore. The dividing line between inward and outward motions connects the centers of divergence. Figure 10 (upper panels) shows color-scaled maps of the velocity magnitudes (ranging from 0 to $1 \mathrm{~km} \mathrm{~s}^{-1}$ ) for the more regular-shaped pores (1 and 2) in our sample, which are represented in the the two upper rows of Fig. 9. Note that both pores exhibit proto-penumbral structures, which are also visible in other pores of the sample. The centers of divergence are clearly identified in the maps of Fig. 10 as black structures around the pores. Another important distinctive feature in these maps is that the highest speeds are located beyond these black structures away from the pore.

In an attempt to study a possible systematic trend in the variation of the speeds around the most regular-shaped pores with approximately the same size in our sample, as a first approach and for simplicity we calculated the mean velocity magnitude in consecutive ribbons (strips) encircling the pores 1 and 2. A total of 12 adjacent ribbons ( $\sim$ '. 24 wide), referred to as ring-like structures surrounding the pore, are used for this analysis.

We emphasize that the following results should not be taken as an attempt to globalize the properties and behavior of the flows around all solar pores, but as a preliminar and simplistic case in which we can compare two pores of our sample under similar conditions (size, shape, polarity among others) and apply the above-mentioned method of computing velocities in ribbons around them. In a future work we will conduct a study where we compare more general cases.

Figure 10 (lower panels) plots the speed versus distance to the pore border. The velocity magnitudes increment as a function of this distance: 1) the mean value of speed increments rapidly at distances ranging from $\sim 0{ }^{\prime} 15$ to $0 . ' 4 ; 2$ ) in the range $0 .{ }^{\prime} 4-$ 1 .' 0 the variation curve is flatter; 3 ) in the range 1 .' $0-1$.' 6 we again obtain very sharp increments. Up to this distance from 

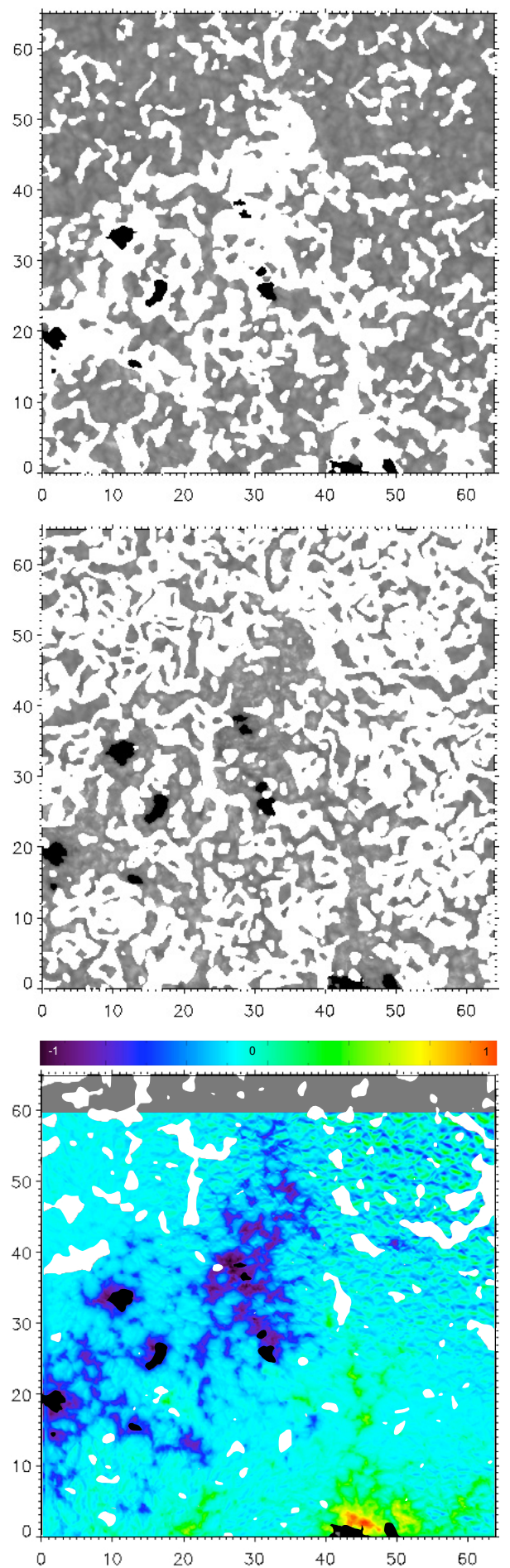

Fig. 7. Location of the areas (in white) where the magnitude of horizontal velocities is lower than $0.3 \mathrm{~km} \mathrm{~s}^{-1}$ (upper panel), between $0.3-0.8 \mathrm{~km} \mathrm{~s}^{-1}$ (middle panel) and greater than $0.8 \mathrm{~km} \mathrm{~s}^{-1}$ (lower panel). Background images in the top and middle panels correspond to a 48-min average image (SST G-band series 1, see Table 1), and in the bottom panel to a false-color normalized (factor of $2300 \mathrm{G}$ ) magnetogram of the region. Pores are colored in black. The coordinates are expressed in arcsec.

the pore border we observe the same behavior in both pores. Nevertheless, the mean speed values at $1^{\prime \prime} 6$ are quite different ( 250 vs. $\left.390 \mathrm{~m} \mathrm{~s}^{-1}\right)$. At further distances $\left(>1^{\prime \prime} .6\right)$ the trend in the mean velocity magnitudes differs substantially. We must bear in mind that the flows around the pore can also be affected by the
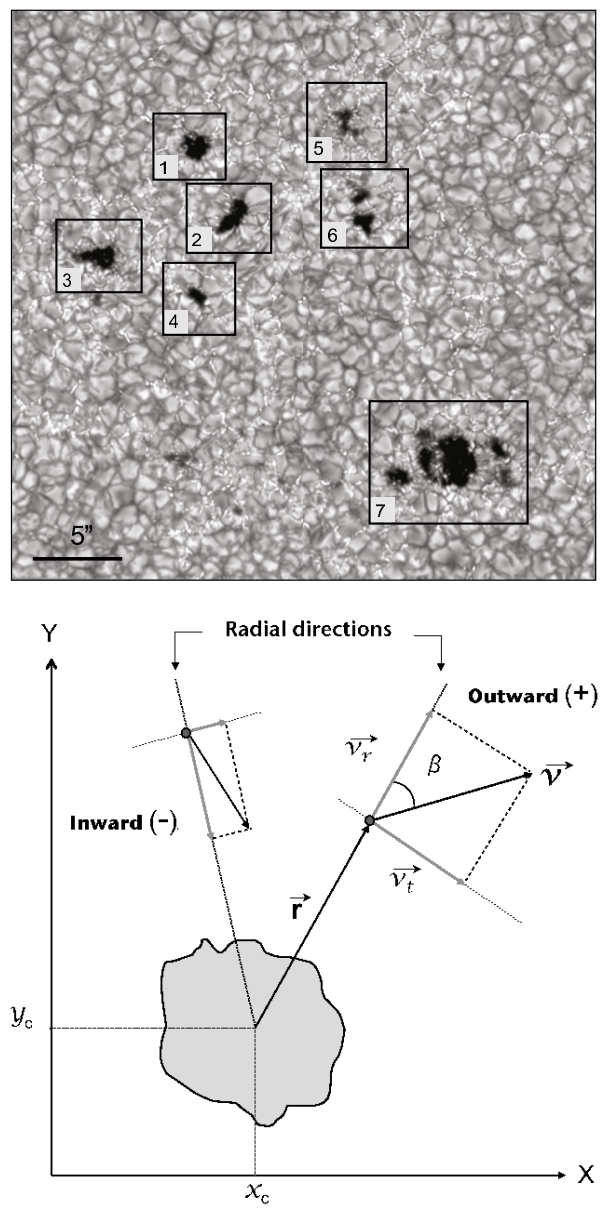

Fig. 8. Upper panel: emerging active region observed with the SST (G-band) on 2007 September 30. Lower panel: sketch showing the projection applied to the velocities around a solar pore centered at $\left(x_{\mathrm{c}}, y_{\mathrm{c}}\right)$ with respect to the orthogonal coordinate system $X, Y$. The figure shows the velocity vectors $v$ for two points in the granulation region around the pore (small black dots). The projection of $\boldsymbol{v}$ along the radial and transversal directions shows the radial $v_{\mathrm{r}}$ and transversal $v_{\mathrm{t}}$ velocity components, respectively.

intrinsic characteristics of every single pore and by the contribution from other sources in the neighbourhood, e.g. pores in the vicinity.

\section{Conclusions}

The proper motions in solar active regions displaying pores are analyzed from high-resolution time series of images. The observing material stems from coordinated ground-based and space observations. Thus part of this material was acquired with the Swedish 1-m Solar Telescope and reconstructed with the novel MFBD and MOMFBD techniques to achieve image resolutions near the diffraction limit. The other part of the data stem from the solar telescope on board the Hinode satellite. The long duration, stability and high-resolution of the time series achieved by Hinode enable us to study dynamical properties of the photospheric horizontal flows along periods of time much longer than those typically possible from ground-based observations, which are restricted by varying seeing conditions.

The local correlation technique applied to the time series allowed us to track the proper motions of structures in solar active regions and particularly in the areas nearby solar pores. Proper motions have been tracked in a variety of active regions for 

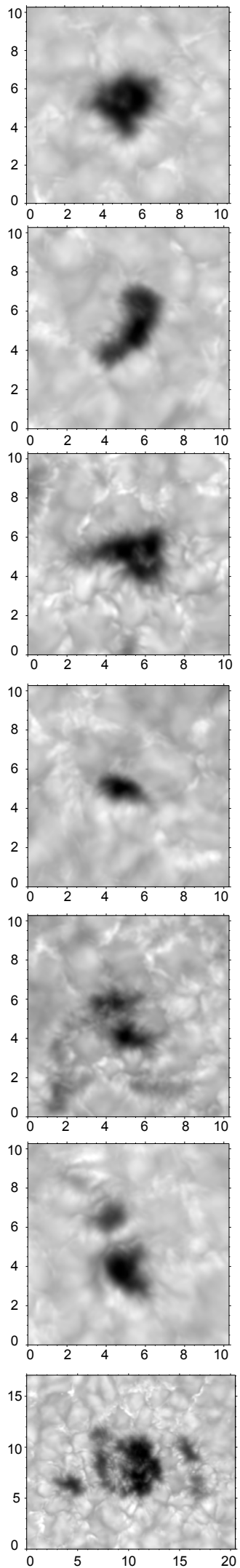
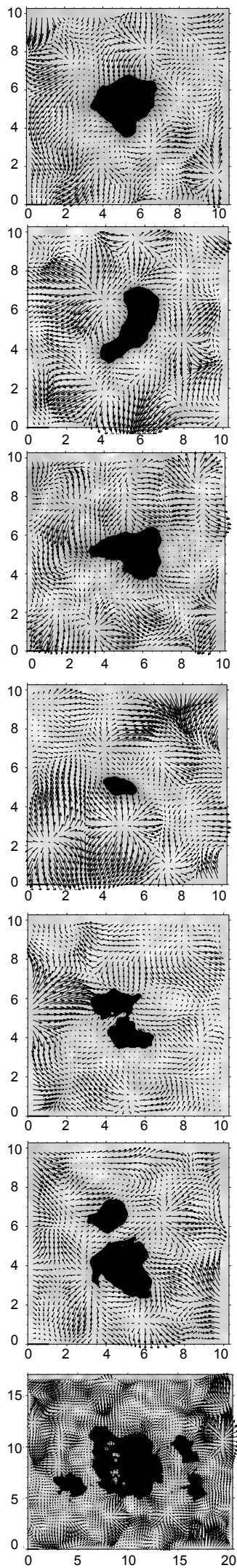
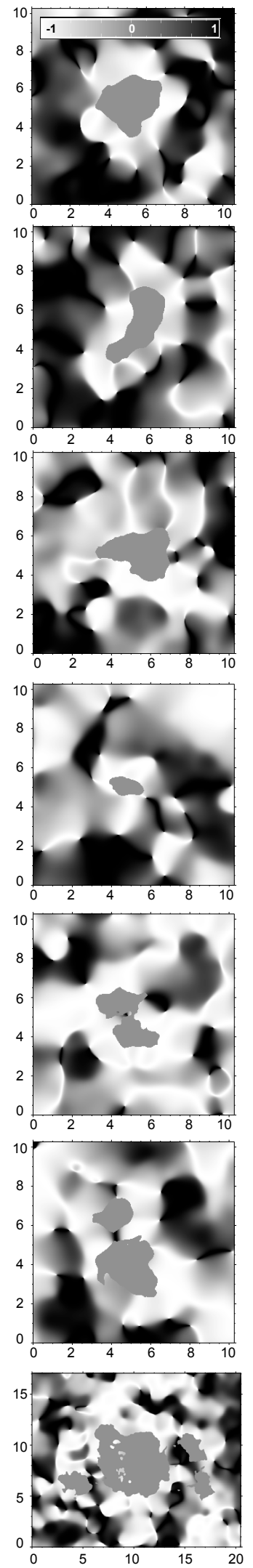
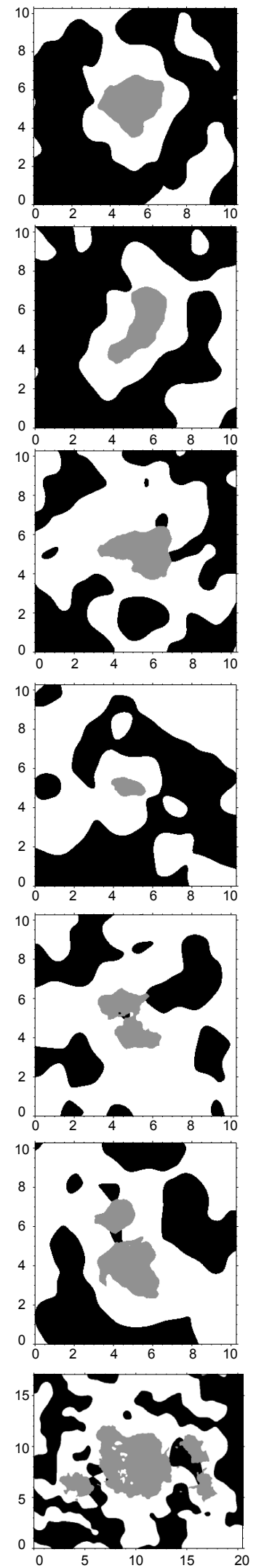

Fig. 9. Distinction between inward and outward motions surrounding solar pores displayed in Fig. 8 (upper panel). The rows display pores from 1 to 7 (top to bottom) and the four columns correspond to (left to right) the average image of the time series, the map of horizontal velocities, the map of $\cos \beta$ and the binary map of inward (white) and outward (black) radial velocities. The length of the black bar at coordinates $(0,0)$ in the map of horizontal velocities corresponds to $1.6 \mathrm{~km} \mathrm{~s}^{-1}$. The gray bar in the top $\cos \beta$ map shows the scale for the cosine from $-1\left(\beta=180^{\circ}\right)$ to $1\left(\beta=0^{\circ}\right)$. See Fig. 8 (lower panel) and the text for details. The spatial units are in arcsec. 

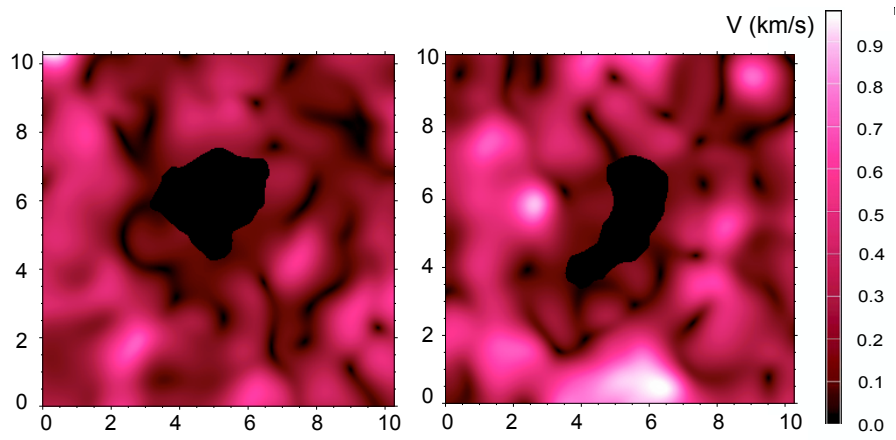

authors describe, which are not in the immediate surroundings of the pore but are separated by the annular inward motion, would then correspond not to moat flows, but to the outward flows originated in the regular mesh of divergence centers around the pore.

Acknowledgements. The Swedish 1-m Solar Telescope is operated in the island of La Palma by the Institute of Solar Physics of the Royal Swedish Academy of Sciences in the Spanish Observatorio del Roque de los Muchachos of the Instituto de Astrofísica de Canarias. We thank the scientists of the Hinode team for the operation of the instruments. Hinode is a Japanese mission developed and launched by ISAS/JAXA, with NAOJ as domestic partner and NASA and STFC (UK) as international partners. It is operated by these agencies in cooperation with ESA and NSC (Norway). The reconstruction of images using the MOMFBD technique is a very computationally expensive task. To reduce the time needed for it, we made use of the Condor workload management system (http://www.cs.wisc. edu/condor/). S. Vargas thanks the people from the Institute of Solar Physics in Stockholm for his support during his short stay in Sweden. Valuable comments of the referee and the editor helped to improve the manuscript greatly. Partial support by the Spanish Ministerio de Ciencia e Innovación through projects ESP2006-13030-C06-01 and AYA2007-63881, and financial support by the European Commission through the SOLAIRE Network (MTRN-CT-2006-035484) are gratefuly acknowledged.

\section{References}

Fig. 10. Analysis of the velocity magnitudes of proper motions around the first to regular-shaped solar pores in Fig. 9. Upper panels: magnitude of horizontal velocities in false-color representation. Lower panels: plot of the mean velocity magnitude versus the distance from the pore border. The spatial units are in arcsec.

periods of typically 20-60 min, but one also for several hours. We conclude that the flow patterns derived from different observational sets are consistent among each other in the sense that they show the determinant and overall influence of exploding events in the granulation around the pores and in the whole FOV. Motions toward the pores in their nearest vicinity are the dominant characteristic we claim to observe systematically. Thus we do not find any trace of moat flow in the wide sample of pores studied. The motions at the periphery of the pores are basically influenced by the external plasma flows deposited by the exploding events, as suggested by other authors in previous works (Sobotka et al. 1999; Roudier et al. 2002; Sankarasubramanian \& Rimmele 2003). In addition, the horizontal velocity magnitudes are clearly lower $\left(<0.3 \mathrm{~km} \mathrm{~s}^{-1}\right)$ in the nearest locations surrounding the pores and in general in the more magnetized regions in the FOV, as expected due to the inhibition of convection.

Our results also agree with recently developed $3 \mathrm{D}$ radiative magnetohydrodynamic simulations of pore-like magnetic structures that report downflows surrounding them, maintained by horizontal flows towards the simulated pore (Cameron et al. 2007). Moreover, we interpret the dividing line between radial inward and outward motions found by Deng et al. (2007) outside the residual pore in the last stage of a decaying sunspot, as corresponding to the location of the centers of divergence of the exploding events around the pore. The outward motions these

Cabrera Solana, D., Bellot Rubio, L. R., Beck, C., \& Del Toro Iniesta, J. C. 2006, ApJ, 649, L41

Cameron, R., Schüssler, M., Vögler, A., \& Zakharov, V. 2007, A\&A, 474, 261

Deng, N., Choudhary, D. P., Tritschler, A., et al. 2007, ApJ, 671, 1013

Giordano, S., Berrilli, F., Del Moro, D., \& Penza, V. 2008, A\&A, 489, 747

Hirzberger, J. 2003, A\&A, 405, 331

Keil, S. L., Balasubramaniam, K. S., Smaldone, L. A., \& Reger, B. 1999, ApJ, 510,422

Kosugi, T., Matsuzaki, K., Sakao, T., et al. 2007, Sol. Phys., 243, 3

Löfdahl, M. G. 1996, Ph.D Thesis, Stockholm University, Stockholm, Sweden Löfdahl, M. G. 2002, Proc. SPIE, 4792, 146

Molowny-Horas, R., \& Yi, Z. 1994, ITA (Oslo) Internal Rep. No. 31

November, L. J., \& Simon, G. W. 1988, ApJ, 333, 427

Roudier, Th., Bonet, J. A., \& Sobotka, M. 2002, A\&A, 395, 249

Sainz Dalda, A., \& Martínez Pillet, V. 2005, ApJ, 662, 1176

Sankarasubramanian, K., \& Rimmele, T. 2003, ApJ, 598, 689

Scharmer, G. B., Bjelksjö, K., Korhonen, T. K., Lindberg, B., \& Petterson, B. 2003a, Proc. SPIE, 4853, 341

Scharmer, G. B., Dettori, P. M., Löfdahl, M. G. , \& Shand, M. 2003b, Proc. SPIE, 4853,370

Sobotka, M., Vázquez, M., Bonet, J. A., Hanslmeier, A., \& Hirzberger, J. 1999, ApJ, 511, 436

Thomas, J. H., \& Weiss, N. O. 2008, in Sunspots and Starspots, Cambridge Astrophysics Series, 46 (Cambridge: CUP)

Title, A. M., Tarbell, T. D., Simon, G., \& the SOUP team 1986, Adv. Space Res., 6,253

Tsuneta, S., Ichimoto, K., Katsukawa, Y., et al. 2008, Sol. Phys., 249, 167

Van Noort. M., Rouppe van der Voort, L., \& Löfdahl, M. G. 2005, Sol. Phys., 228, 191

Vargas Domínguez, S. 2008, Ph.D Thesis, University of La Laguna - Instituto de Astrofísica de Canarias, Tenerife, Spain

Vargas Domínguez, S., Bonet, J. A., Martínez Pillet, V., et al. 2007, ApJ, 660, L165

Vargas Domínguez, S., Rouppe van der Voort, L., Bonet, J. A., et al. 2008, ApJ, 679,900

Wang, H., \& Zirin, H. 1992, Sol. Phys., 140, 41

Zuccarello, F., Romano, P., Guglielmino, S. L., et al. 2009, A\&A, 500, L5 